\title{
Bacterial genome engineering and synthetic biology: combating pathogens
}

\author{
Malathy Krishnamurthy@, Richard T. Moore, Sathish Rajamani and Rekha G. Panchal
}

\begin{abstract}
Background: The emergence and prevalence of multidrug resistant (MDR) pathogenic bacteria poses a serious threat to human and animal health globally. Nosocomial infections and common ailments such as pneumonia, wound, urinary tract, and bloodstream infections are becoming more challenging to treat due to the rapid spread of MDR pathogenic bacteria. According to recent reports by the World Health Organization (WHO) and Centers for Disease Control and Prevention (CDC), there is an unprecedented increase in the occurrence of MDR infections worldwide. The rise in these infections has generated an economic strain worldwide, prompting the WHO to endorse a global action plan to improve awareness and understanding of antimicrobial resistance. This health crisis necessitates an immediate action to target the underlying mechanisms of drug resistance in bacteria.

Research: The advent of new bacterial genome engineering and synthetic biology (SB) tools is providing promising diagnostic and treatment plans to monitor and treat widespread recalcitrant bacterial infections. Key advances in genetic engineering approaches can successfully aid in targeting and editing pathogenic bacterial genomes for understanding and mitigating drug resistance mechanisms. In this review, we discuss the application of specific genome engineering and SB methods such as recombineering, clustered regularly interspaced short palindromic repeats (CRISPR), and bacterial cell-cell signaling mechanisms for pathogen targeting. The utility of these tools in developing antibacterial strategies such as novel antibiotic production, phage therapy, diagnostics and vaccine production to name a few, are also highlighted.
\end{abstract}

Conclusions: The prevalent use of antibiotics and the spread of MDR bacteria raise the prospect of a postantibiotic era, which underscores the need for developing novel therapeutics to target MDR pathogens. The development of enabling SB technologies offers promising solutions to deliver safe and effective antibacterial therapies.

Keywords: Synthetic Biology (SB), Multidrug resistant (MDR) pathogens, Antibiotic resistance, Genome engineering, Antibacterial, Quorum sensing, Gene circuits, Pathogenesis, Recombineering, Targetron

\section{Background}

The emergence of multidrug resistant (MDR) pathogenic organisms has become an important national and global health challenge. Importantly, the evolution of bacterial MDR pathogens is rampant and needs immediate countermeasures to limit lasting damage. According to the World Health Organization's (WHO) report in 2014 on global surveillance of antimicrobial resistance, the increased rise in MDR pathogenic bacteria is putting at risk the ability to treat common ailments such as urinary

\footnotetext{
* Correspondence: rekha.g.panchal.civ@mail.mil

Department of Target Discovery and Experimental Microbiology, Division of Molecular and Translational Sciences, U. S. Army Medical Research Institute of Infectious Diseases (USAMRIID), Fort Detrick, Frederick, MD 21702, USA
}

tract infections, pneumonia and bloodstream infections globally, that were readily treatable for decades. In 2015, the 68th World Health Assembly has endorsed a global action plan to improve awareness and understanding of antimicrobial resistance [1]. This plan calls for the development of new medicines, diagnostic tools, vaccines and other interventions to ensure continued treatment and prevention of infectious diseases caused by bacteria. Therefore, there is an urgent need for understanding drug resistance mechanisms in MDR pathogens and targeting these mechanisms (e.g., antibiotic target site mutation, efflux pump for antibiotic expulsion, etc.) to tackle these pathogens. In addition, identification of novel and improved therapeutic small molecules and 
metabolic engineering for the production of these small molecules is another approach to assuage drugresistance in MDR pathogens.

Recent advancements in synthetic biology (SB) have enabled the development of novel genome engineering tools for the manipulation of microbial genomes for various biotechnological and biomedical applications [2-6]. SB offers a novel platform to bridge the gap between basic and translational research and has the potential for providing innovative solutions to combat infectious agents. SB is an emerging field which combines engineering principles and biological parts to design novel, modular and tunable gene products or genetic circuits for modification of existing biological systems. In addition to building gene circuits for desired cellular function or metabolic engineering, there is a growing interest among synthetic biologists to develop microbial genome engineering tools. Precise changes in the bacterial genome have resulted in the creation of useful biological traits in engineered strains. The marriage of genome engineering tools and SB has further enabled the use of engineered bacteria to address some of the global challenges spanning renewable energy to global health. In particular, the recent advances in bacterial genome engineering methods that can target broad range of bacterial hosts has opened new avenues for fighting bacterial infections [7, 8]. The development of new SB tools should pave the way for developing novel approaches to address the imminent threat with antibiotic resistance in bacteria.

SB applications in bacteria have broadly ranged from building small gene circuits for a desired gene/pathway function to engineering the whole genome [9-12]. A number of investigations employing SB have also offered insights into antibiotic resistance mechanisms. For instance, to delineate the mechanism of resistance to a particular antibiotic, lethal concentrations of antibiotic triclosan was used in Escherichia coli to identify the gene candidates that were involved in triclosan resistance [13]. An overexpressed genomic library was generated in triclosan enriched media and using a DNA microarray, the genes that enabled the growth of $E$. coli in the presence of triclosan were identified and validated by overexpressing the candidate gene in bacteria [14]. Unlike traditional methods, which involve genome sequencing to identify potential genes that confer antibiotic resistance, this approach utilized genome libraries cloned into plasmids for expression in bacteria and enrichment in the presence of antibiotic. Such SB approach allows for genome wide screening and identification of genes as well as the effect of overexpression of these genes on cellular fitness. This is particularly useful for understanding the complex mechanisms of antibiotic resistance and for identifying one or multiple gene targets that lead to resistance. Similarly, SOS response systems in $E$. coli subjected to other antibiotics have been examined by building gene circuits in E. coli to study DNA damage and to understand the role of these systems in antibiotic resistance [15].

Minimal bacterial genomes have been synthesized using top-down and bottom-up approaches for identifying the essential genes in bacteria (E. coli, Pseudomonas putida, Mycoplasma) for potential therapeutic targeting [16-18]. The growth potential and the impact of SB in countering antibiotic resistance are clearly evident from these examples. The focus of this review is to highlight the applications of bacterial genome engineering and synthetic biology tools in targeting emergent bacterial pathogens and further discuss the utility of SB in advancing novel antibacterial therapeutics.

\section{Main text}

\section{Genome engineering tools and their applications for} countering bacterial infections

A number of methods have been developed for engineering bacterial genomes with varying degrees of efficiency, specificity and broad host applicability [19]. Most often, the bacterial genome editing is carried out to knock-out genes, knock-in genes or introduce mutations in the bacterial genome. Though most of these methods were developed in E. coli, in the last decade there has been a rapid development and expansion of these tools to a broad range of bacterial hosts (Fig. 1a). Noteworthy is the tractability of these engineering tools in other pathogenic bacteria, paving the way for exploration and understanding of these pathogens for combating bacterial infections. A number of useful reviews have also detailed the principles and techniques of bacterial genome engineering tools $[7,8]$. The most common tools that are currently being utilized for genome engineering of pathogenic bacteria are summarized in Table 1 and their potential applications in countering bacterial infections are discussed below.

\section{Insight into bacterial virulence, resistance mechanism and biomolecular targets}

Bacterial chromosomal modifications have greatly aided in better comprehension of bacterial pathogenesis and virulence mechanisms. Among the genome engineering methods, the utilization of the $\lambda$-Red recombinase system for insertions, deletions or point mutations of the genome has been very popular. Pioneered by Murphy [20] and later modified by Datsenko and Wanner [21], this method involves the introduction of single- or double-stranded DNA with chromosomal homology regions for recombination [22]. Since its conception, this editing strategy has been made more efficient by modifications to the method developed by Wanner [23-25]. This has also been readily adapted to pathogenic 


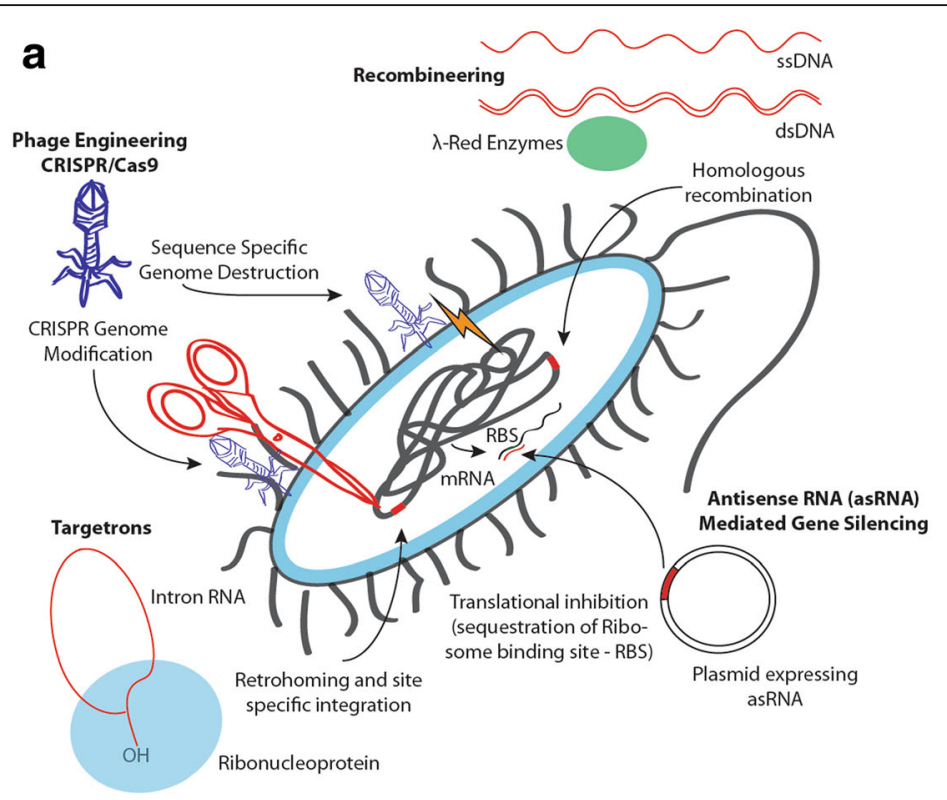

b

Multiplex Genome Engineering (MAGE, portMAGE, etc.)

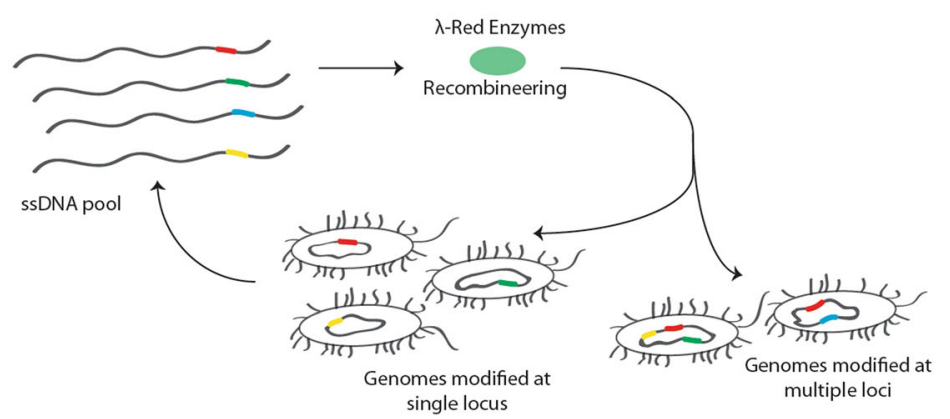

Fig. 1 a) Schematic of genome engineering tools developed in E. coli that have been expanded to broad bacterial hosts b) Multiplexed Automated Genomic Engineering (MAGE) for modifying bacteria at multiple genomic loci

Table 1 Tools available in pathogenic Gram-negative and Gram-positive bacteria for genome modification

\begin{tabular}{|c|c|c|}
\hline Engineering tool & Methodology & Engineered pathogenic strains \\
\hline Recombineering & $\begin{array}{l}\text { Homologous recombination of linear } \\
\text { DNA utilizing } \lambda \text {-Red enzymes Gam, } \\
\text { Exo and Bet }\end{array}$ & $\begin{array}{l}\text { Salmonella enterica [26] }{ }^{a} \\
\text { Escherichia coli [23] }{ }^{a} \\
\text { Pseudomonas aeruginosa [29] }{ }^{a} \\
\text { Streptomyces coelicolor [28] } \\
\text { Shigella dysenteriae }[50]^{a}\end{array}$ \\
\hline PORTMAGE & $\begin{array}{l}\text { Portable Multiplex Automated Genome } \\
\text { Engineering (MAGE) } \\
\text { Utilizing recombineering }\end{array}$ & Salmonella enterica $[35]^{a}$ \\
\hline Targetrons & $\begin{array}{l}\text { Retrohoming of Mobile Group II Introns by } \\
\text { reverse splicing and insertion in genome }\end{array}$ & $\begin{array}{l}\text { Clostridium perfringens [38] }{ }^{\mathrm{b}} \\
\text { Vibrio Cholera [78] } \\
\text { Yersinia pseudotuberculosis [79] }{ }^{\mathrm{a}} \\
\text { Staphylococcus aureus [80] }\end{array}$ \\
\hline $\begin{array}{l}\text { Phage Engineering } \\
\text { CRISPR/Cas9 }\end{array}$ & $\begin{array}{l}\text { Delivery of CRISPR genes and RNA guides } \\
\text { for sequence specific antimicrobials }\end{array}$ & $\begin{array}{l}\text { Carbapenem-resistant Enterobacteriaceae } \\
\text { Enterohemorrhagic Escherichia coli }[55]^{\text {a }}\end{array}$ \\
\hline Antisense RNA & Post-transcriptional gene silencing & $\begin{array}{l}\text { Staphylococcus aureus [81] }{ }^{\mathrm{b}} \\
\text { Streptomyces coelicolor [82] }\end{array}$ \\
\hline
\end{tabular}


bacterial strains for investigating the roles of genes in pathogenesis [23, 26-29]. Pseudomonas aeruginosa, an opportunistic pathogen that causes nosocomial infection and chronic infections of cystic fibrosis lungs, utilizes two major quorum sensing systems LasR/LasI and RhlR/ RhlI for orchestrating the production of virulence factors and biofilm formation [30]. To understand their roles in $P$. aeruginosa virulence, $\lambda$-Red recombination was successfully used to generate $\Delta l a s R$ mutant and $\Delta l a s R /$ $\triangle r h l R$ double mutant $P$. aeruginosa strains to delineate their functions [31]. Using Caenorhabditis elegans 24h fast-kill infection assay, it was shown that $C$. elegans was more rapidly killed by wild-type and $\Delta l a s R$ mutant compared to the $\Delta r h l R$ mutant or $\Delta l a s R / \Delta r h l R$ double mutant strains, indicating that RhIR function is important for virulence. The authors further identified a small molecule called meta-bromo-thiolactone (mBTL), an analog of $\mathrm{N}$-acyl-L-homoserine lactone (AHL), a native $P$. aeruginosa cell-cell signaling molecule, that was shown to attenuate pyocyanin and biofilm production in the wildtype strain. To identify if RhlR or LasR receptor is the molecular target for $\mathrm{mBTL}, \lambda$-Red recombination system was used to generate $\Delta l a s R$, and $\Delta l a s R / \Delta r h l R$ double mutants to demonstrate that RhlR is the relevant in vivo target of mBTL.

One of the key adaptions of Salmonella enterica serovar typhirium, a pathogen that causes typhoid in humans, is its ability to survive inside host phagocytes. Macrophages express inducible nitric oxide (NO) synthase (iNOS) in response to lipid A, fimbriae and porins that decorate the Salmonella envelope. It has previously been demonstrated that NO produced as an innate response by macrophages can impact amino acid biosynthesis in Salmonella by targeting DksA. DksA is a key RNA polymerase regulatory protein in Salmonella, which has been implicated in the bacterium's resistance. To determine if DksA is responsible for the antinitrosative defenses in Salmonella, Henard and Vazquez-Torres generated a $d k s A$ mutant using $\lambda$-Red recombination $[32,33]$. They determined that $\triangle d k s A$ mutant Salmonella strains are hypersusceptible to the bacteriostatic effects of $\mathrm{NO}$ and were noticeably attenuated in its infectivity as shown in a murine model of acute systemic infection.

In order to make genome editing high-throughput, a recombineering method called Multiplex Automated Genome Engineering (MAGE) has been developed recently to replace the conventional $\lambda$-Red recombinase based recombineering method (Fig. 1b). This method employs $\lambda$-Red recombinase system and a pool of oligos to rapidly introduce simultaneous modifications in the E. coli genome within days [34]. Further improvements to this method, designated pORTMAGE (portable MAGE) have been made and extended to clinically relevant strains such as Salmonella enterica [35]. Using
pORTMAGE, ten antibiotic resistance mutations were introduced simultaneously in the genome of $S$. enterica and $E$. coli to study the extent of conservation of molecular mechanisms of antibiotic resistance amongst these bacteria.

For bacteria that are intractable to common engineering methods such as the $\lambda$-Red recombinase system, mobile group II introns have been utilized for site specific editing of the genome. Mobile group II introns are bacterial retrotransposons that contain an intron RNA and an intron-encoded reverse transcriptase. The mobile group II introns are ribozymes that can insert into specific targets by the process of retrohoming [36]. Using predictive algorithms, the intron RNA can be redesigned to form a 'targetron', such that a target DNA site of choice can be edited. This method has been adapted to a number of pathogenic strains for understanding mechanisms of virulence [37]. For several medically relevant Clostridium species which are recalcitrant to recombinations, a targetron based method named ClosTron technology has been developed for successful genome editing [38]. In one example, ClosTron technology was used for site-directed mutagenesis of a germination specific protease called CspC in Clostridium difficile, a causative agent of foodborne infection and diarrhea. This study helped determine the role of CspC in host bile acid recognition for in vivo germination and disease establishment [39].

In another example, the targetron technology was used in studying virulence mechanism in Pasteurella multocida, an animal pathogen that causes fowl cholera in wild birds and poultry, hemorrhagic septicemia in ungulates and atrophic rhinitis in swine. The polysaccharide capsule that is composed of hyaluronic acid is a major virulence factor. To investigate the mechanism of capsule formation and validate the role of global transcriptional regulator Fis in capsule formation, Steen and coworkers used the targetron technology in Pasteurella to generate Fis mutants [40]. They determined that not only is functional Fis protein required for capsule formation, but it is also required for regulation of number of virulence genes.

\section{Production of novel antibiotics}

The rate at which bacteria are developing resistance to existing antibiotics is alarming and warrants our immediate attention to the development of novel antibiotics for combatting bacterial pathogens. Typically, antibiotics are naturally derived small molecules that are produced by genetically encoded pathways. They represent a rich source of chemical diversity and are produced by an array of microorganisms. The use of genome editing tools to engineer new biosynthetic pathways in microbial hosts is proving to be an ideal strategy for production of 
novel antibiotics [41]. In one instance, Eustaquio and co-workers employed the $\lambda$-Red recombinase methodology to inactivate clo-hal and cloz genes in the biosynthetic gene cluster which produces the antibiotic clorobiocin, a bacterial DNA gyrase inhibitor. The mutated cosmid bearing the inactivated clo-hal cassette or cloZ gene was introduced in $S$. roseochromogenes to study the functional role of these genes in chlorination of the molecule. Furthermore, this strain was then used for producing an analog of clorobiocin, which has a methyl group instead of chlorine substitution and showed reduced antibiotic potency [42].

The $\lambda$-Red recombinase based recombineering method has also been applied for combinatorial biosynthesis of daptomycin (Cubicin), an antibiotic approved in the US for the treatment of skin infections caused by Grampositive Staphylococcus aureus [43]. The antibiotic also has potent in vitro bactericidal activity against methicillin resistant S. aureus (MRSA), penicillin-resistant Streptococcus pneumoniae (PRSP), vancomycin-resistant enterococci (VRE), and vancomycin-resistant S. aureus (VRSA) [44]. With the emergence of bacterial resistance to this antibiotic, there has been an interest to make second generation derivatives of daptomycin. Using a novel approach, the $\lambda$-Red recombinase methodology has been used for exchanging multiple modules in the subunits of the nonribosomal peptide synthetase (NRPS) in the daptomycin biosynthetic pathway using $E$. coli as a heterologous host [45]. The combinatorial biosynthesis approach was used to generate a library of novel lipopeptides with modifications of the core peptide, of which some compounds were as active as daptomycin. The above examples illustrate the huge potential of bacterial genome engineering tools in biosynthesis of novel antibiotics.

\section{Attenuated vaccine strain development}

One of the effective strategies to prevent infections is the use of vaccination, which establishes an immunological memory of a foreign agent by triggering the body's innate immune response. Inactivated or attenuated vaccines from the actual pathogen have been successfully used in the past for defense against bacterial infections. Attenuated live vaccines are created by decreasing the virulence of the pathogen to weaken their infection potential without compromising the robust host immune response that is required for protection during future infections that could be caused by the same pathogen (For example: MTBVAC against Mycobacterium tuberculosis [46], Ty21a against Salmonella typhi [47]). There is a growing focus on using recombinant DNA technology for producing attenuated strains of pathogenic bacteria [48]. Genome engineering tools are now being routinely explored for the possibility of reducing bacterial virulence. For example, Ranallo and coworkers have successfully demonstrated the extension of the $\lambda$ - Red recombination method in Shigella for the development of live vaccine strains [49]. They utilized over 13 different isogenic strains to delete genes involved in various functions such as intracellular growth and survival (asd), cell to cell spread (virG), invasion (ipaB), enterotoxic activity (set $1 A$, sen) to name a few. Utilizing a plaque assay, they determined that the virG deleted strain reduced plaque formation significantly. In further virulence testing using the keratoconjuctivitis model (Sereny test), they found that only the virG deleted strain was attenuated.

More recently, Salehi and cowokers validated the utilization of this recombineering technique for the production of live attenuated Shigella dysenteriae strain by deleting ipaD gene [50]. The ipaD is a chaperonin protein and part of the type III secretion system which secretes invasion plasmid antigen (Ipas) proteins that are responsible for Shigella penetration and invasion into epithelial cells. The authors hypothesize that the deletion of ipaD gene could potentially inhibit secretion of IpaD, $\mathrm{IpaB}$ and IpaC proteins and thereby suppress Shigella invasion.

The targetron methodology has also been used to generate candidate vaccine strains in pathogens where the $\lambda$ - Red recombination method works poorly. Combining the targetron methodology discussed above and the well-known Cre-lox recombinase system, a vaccine strain of the Gram-positive pathogen Staphylococcus aureus was generated by a novel approach called Genome Editing via Targetrons and Recombinases (GETR) [37]. The researchers generated introns that could integrate lox sequences upstream and downstream of the 15-kb Staphylococcus aureus pathogenicity island I (SaPI-1). The lox sites are specific DNA sequences that can be targeted by the Cre recombinase enzyme. The expression of Cre recombinase resulted in Cre-mediated recombination that deleted the intervening region in the SaPI-1 leading to the generation of a vaccine strain. The application of such techniques in clinical isolates of Staphylococcus can be very useful in generating vaccine strains for MDR strains for overcoming the antibiotic resistance challenge. Though the safety and efficacy of these vaccines need to be assessed periodically, the above examples highlight the power of genome editing tools for their potential to design live vaccines. Development of promising and safe to use vaccines will have broad applications in preventive healthcare and will be a stepping stone for the development of oral vaccines in other pathogenic bacteria [51].

\section{Specificity in pathogen killing and pathogen detection for diagnosis}

Tools adapted from bacteriophage assist in understanding host-pathogen interactions and serve as targeted 
therapeutics [52-54]. Phage can specifically kill virulent strains of bacteria that bear very close sequence alignment to harmless strains via the use of the clustered regularly interspaced short palindromic repeats (CRISPR)/Cas9 system. The CRISPR/Cas9 system specifically targets a DNA sequence for double strand break formation, resulting in death of the bacteria. Phage can deliver RNA guides with CRISPR associated proteins (Cas) to pathogenic bacteria [55, 56].

Towards targeted therapies against pathogenic bacteria, Citorik et al. have demonstrated sequence specific antimicrobials [55], which overcome extremely high genome sequence similarity between non-pathogenic and pathogenic strains by targeting small sequence variations present in the pathogenic strain. It is possible to harness the ability of Cas to target specific sequences and differentiate between a little as one mismatch between target and non-target genomic DNA to kill the pathogenic bacterial population [55]. In the model system of Galleria mellonella larvae, targeted nucleases showed greater antimicrobial activity than antibiotic chloramphenicol. The RNA guided DNA nucleases targeting of the enterohemorrhagic $E$. coli intimin virulence gene and the nuclease activity at this locus proved toxic to the pathogenic bacteria [55].

In another application of the CRISPR system, Yosef et al. designed a novel two phage CRSIPR system consisting of temperate and lytic phage programmed to specifically sensitize and kill antibiotic resistant bacteria [57]. Initially, lysogenic phage carrying CRISPR machinery was used to target the antibiotic resistance genes and confer lytic phage resistance to these cells. The cells became sensitive to antibiotic but were resistant to lytic phage. In the second step, lytic phage was used to kill any remaining antibiotic resistant cells thus enriching the population of the antibiotic sensitive cells, which can then be killed with antibiotics. The authors propose that this strategy can be very useful for treating hospital surfaces or for skin surfaces of medical personnel.

Phage can also serve as diagnostic and detection tools for infection yet do not require amplification of the host bacteria as the phage population increases during infection. As the phage infects specific bacteria, the phage genomic template becomes enriched in the population and targeted bacteria can be killed by the phage. A quantitative PCR following phage infection can indicate amplification of phage DNA infecting a specific bacteria [53]. Diagnostic phages exist for highly pathogenic bacteria, such as phage phi A1122 for Yersinia pestis, as well as phage reporter systems for Bacillus anthracis and Mycobacterium tuberculosis [58].

\section{Application of synthetic biology for targeting bacterial infections}

Key advances in precision genome engineering have resulted in the development of a toolbox that is proving highly valuable for redesigning microbial genome structure for useful applications. The bacterial genome engineering strategies discussed in the previous sections clearly illustrate the utility of these tools in synthetic biology applications for targeting infectious diseases. Apart from gene insertions, deletions or mutations for modification of the genome using engineering tools, one of the main goals of SB is to build and integrate gene circuits which process signals within a living cell for a desired output. Gene circuits have been assembled in microbes using biological parts or functional units for various biomedical applications $[59,60]$. Modular biological parts can be connected to develop circuits based on electrical engineering principles with input and output responses that can be analog or digital. Using this engineering framework, SB has potential applications in biofuel production, synthesis of industrial chemicals or natural product substitutes, biomedical applications or understanding and countering bacterial infections (Fig. 2a) $[2,5,59]$. In the following section, we highlight few examples of regulatory biological components that have been utilized for biomedical applications focused on bacterial infections.

\section{Bacterial quorum sensing based circuits}

Bacteria effectively sense and respond to environmental signals as part of their natural survival and proliferation strategies. SB has harnessed these mechanisms to sense and respond to clinically relevant signals. The development and engineering of bacterial small molecule signaling mechanism based SB circuits for targeting bacterial pathogens has also been used as a novel approach in design of circuits.

Bacterial cell population-density dependent behavior termed quorum sensing (QS) is a highly evolved natural signaling circuit found in bacteria [60]. It involves small molecule signal production and sensing by the native bacterium via their cognate signal receptor that then modulate the expression of the target genes [60]. Initial QS SB circuit engineering in bacteria was demonstrated by Weiss and Knight Jr. [61]. One of the Vibrio fischeri QS systems relies on a key small molecule signal $\mathrm{N}$ Acyl-L- homoserine lactone (AHL) for its bioluminescence production. In their first SB circuit, AHL synthesis catalyzed by luxI (AHL synthase) and the AHL signal receptor luxR were engineered into two separate populations (A and B populations respectively) of E. coli. When these strains were cocultivated, it was observed that the AHL signals produced by E. coli (luxI) (population A) freely diffused out the cell and bound to its cognate LuxR receptor in E. coli (luxR) subpopulation (population B). The activated LuxR-AHL complex in turn activated luxI::gfp promoter-reporter fusion resulting in GFP production [61]. With this early demonstration, interest in the 
a

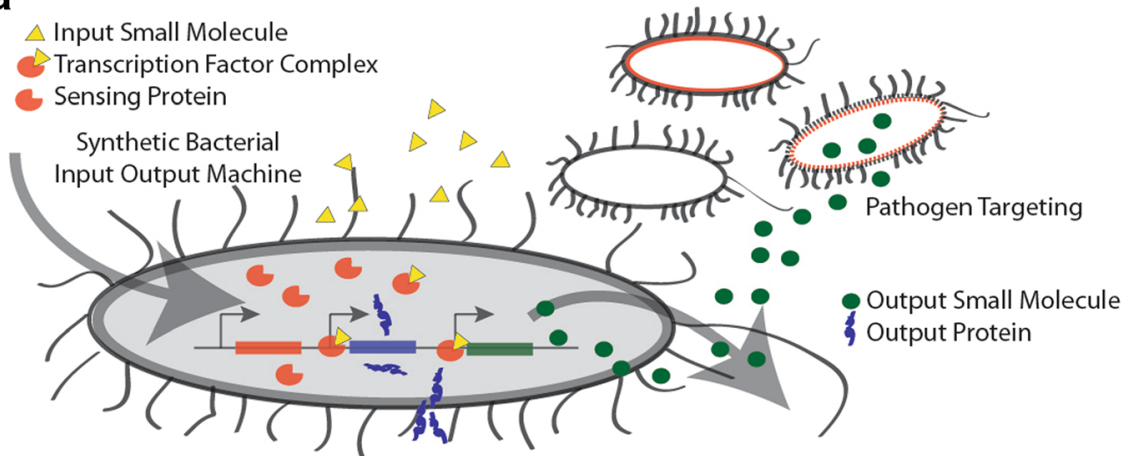

b
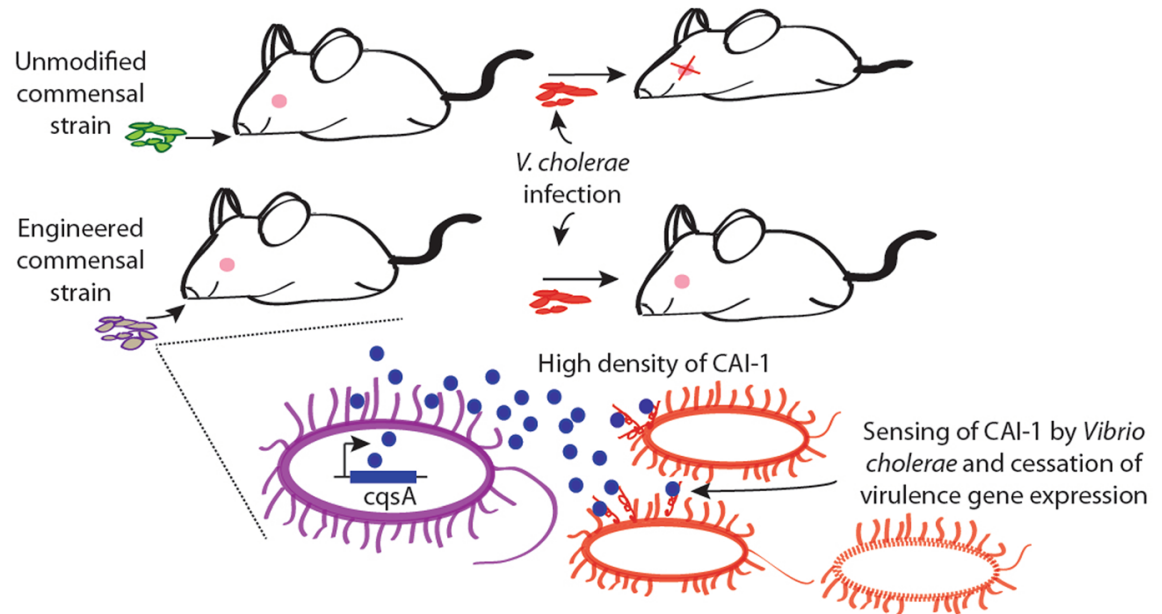

Fig. 2 a) Synthetic biology circuits can be engineered with desired input and output signals to kill bacterial pathogens; b) Engineering probiotic bacteria that expresses QS molecule Cholerae autoinducer-1 (CAI-1) to target Vibrio cholerae infection in a mouse model [65]

utility of QS circuits in SB has increased in a number of biomedical applications including diagnostic tools, cancer, immune diseases, metabolic disorders, infectious disease therapies, drug production through fermentation, biosensing, etc [62-64].

In an important example, Duan and March showed that feeding infant mice with engineered probiotic $E$. coli to constitutively overexpress Vibrio cholerae QS signal (S)-3-hydroxytridecan-4-one or Cholerae autoinducer-1 (CAI-1) that down-regulates biofilm production substantially increased the mice survival rate from $V$. cholerae infections $(92 \%$ with 8 h pretreatment) [65] (Fig. 2b). Using a gene circuit, Saeidi et al. engineered $E$. coli with $P$. aeruginosa LasR AHL receptor to sense $P$. aeruginosa AHL signal $\mathrm{N}$ - 3-oxododeconoyl-L-HSL and auto-regulate the activation of killing and lysis gene products (E7 lysis protein and Pyocin S5) that targeted $P$. aeruginosa [66]. In conjunction with this design, other QS circuits for pathogen targeting based on detection, destruction and secretion modules have been successfully engineered into useful bacteria. For example, in a proof-of-concept study, pathogen $P$. aeruginosa elimination was demonstrated using programmed $E$. coli sensing $P$. aeruginosa $\mathrm{QS}$ signal N-3-oxododeconoyl-L -HSL and activating the production and secretion of chimeric lethal protein bacteriocin CoPy [67].

Another example of this modular bacterial QS circuit engineering was demonstrated by programming $E$. coli to seek and kill $P$. aeruginosa. This system utilized CheZ, a motility promoting protein and two engineered secrete and kill proteins DNaseI and MicrocinS that promoted biofilm disruption and lethality [68]. Most of these QS circuits essentially target Gram-negative bacterial circuits and in particular only the AHL class of QS molecules. There is a great diversity of QS molecules (non-AHL classes) and pathways that still remain underexplored for targeting and can be effectively used for pathogen control.

\section{RNA based circuits}

RNA based biological parts called riboregulators are also gaining attention in SB circuit design due to their tunable and modular nature. These RNA hairpin tools are designed to sequester the ribosome 
binding site (RBS) upstream of the start site of the mRNA encoding a gene in order to block translation [58, 69-72]. In one application of these tools, RNA switches called "toehold" switches developed by Pardee et al. have been used for diagnostic application for in vitro, cell-free, paper-based devise for sensing Ebola mRNA and mRNAs of antibiotic resistance genes [73]. RNA based diagnostic gene network consists of a reporter gene network ( $g f p$, lacZ), where the RBS is sequestered upstream by the toehold switch. The gene network and a cell-free coupled transcription/translation system are freeze dried on a paper or other porous material and can be activated by rehydration with the test sample which consists of the mRNA to be detected. Messenger RNA sensors for antibiotic resistance genes, upon sensing the target gene, showed significant induction of reporter gene making this tool highly promising and costeffective in the detection and diagnosis of bacterial infections in clinical samples.

Apart from application in diagnostics, the ability of fine-tuning gene expression and the modular nature of riboregulators make them excellent candidates for chromosomal integrations using existing genome editing tools for biosynthetic pathway engineering in bacterial hosts. It is evident from the above examples that genome engineering and synthetic biology tools in bacteria can have significant impact on a number of applications for targeting bacterial infections (Fig. 3).

\section{Conclusions}

The integration of engineering principles and biology in the last decade has opened up new avenues for development of novel therapeutics in treating diseases. The rapid emergence of bacterial antimicrobial resistance and limited success in identifying new antibiotics warrants the identification and validation of novel bacterial drug targets. SB driven genome editing techniques offer new avenues to pursue bacterial target identification and present the possibilities for development of novel antimicrobial therapeutics. The availability of high-throughput bacterial genome editing tools coupled with advancement in DNA synthesis technologies provides new opportunities for metabolic engineering of large gene clusters in microbial hosts. By rational combination of gene parts, biosynthesis can be potentially reprogrammed for generation of novel small molecules for therapeutic purposes [48]. This is further facilitated by in silico whole genome mining and software algorithms that predict the gene clusters which can be used in biosynthetic pathway engineering for production of novel antibiotics $[48,74]$. This offers huge potential for combinatorial biosynthesis of natural product analogs for the discovery of novel antibiotics, as in the example of antibiotic daptomycin described in the earlier section [41].

In addition to antibiotics, development of alternative treatment options employing bacteriophage and probiotic bacterial engineering for pathogen targeting and destruction are critical for the fight against infections, including the drug-resistant bacterial pathogens. Advances in computational gene circuit design coupled with improvements in large scale DNA synthesis heralds a new era of SB based therapeutic approaches. It is now possible to design whole bacterial genomes from synthesized components. Viewing genetic code as an analog to computer software, one can then "boot" the synthetic genome in a compatible cellular environment [75]. Additionally, computational design of synthetic circuits

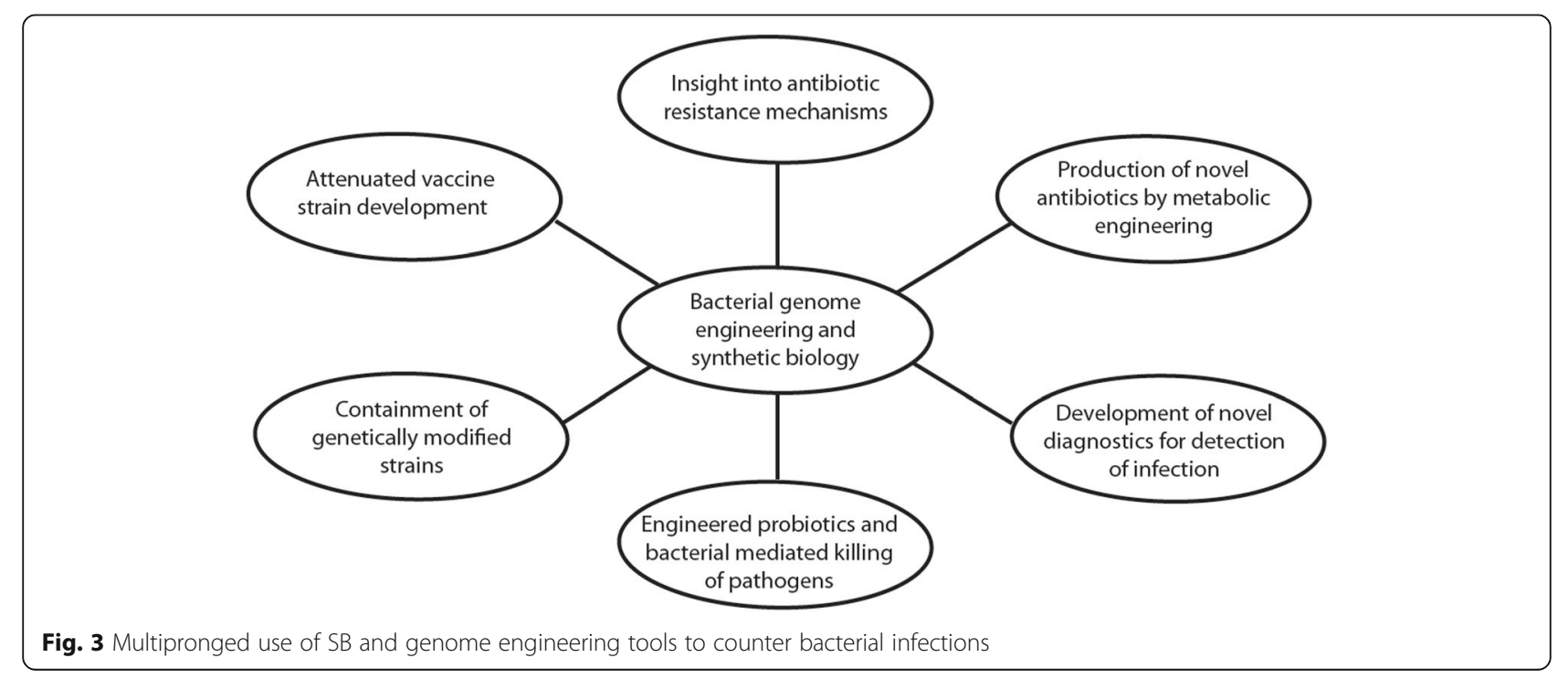


will be highly useful for predicting the optimal combination of synthetic parts for a desired cellular function. Recently, Voigt and coworkers developed the Cello software, which allows a user to program a desired circuit function in E. coli and compile the code into a DNA sequence for synthesis [76]. Computer-aided design (CAD), high-throughput DNA synthesis and advanced genome editing techniques will prove highly valuable for generating re-programmed bacterial strains for therapeutic applications. The combination of advanced computational methods for predictive SB systems, and rapid progress in technologies for efficient large scale DNA synthesis as well as high-throughput, automated genome engineering such as MAGE, pORTMAGE indicates that SB has immense potential to deliver novel solutions for pathogen control.

Genetically modified bacteria offer great hope for finding novel solutions to detect and treat infections. However, it is also imperative to periodically asses the biosafety of these organisms to avoid accidental release of synthetic bacteria generated in some of these applications. To address this concern, two engineered safeguard systems called the 'Deadman' and 'Passcode' kill switches have been developed by Collins and colleagues in E. coli [77]. These switches are based on circuits that need specific input(s) of small-molecules for cell survival. In the absence or presence of the specific molecules, toxin gene expression is activated leading to cell death. The kill switch circuit designs can potentially be incorporated in a broad range of bacterial hosts to ensure safe handling of these modified organisms. It is clearly evident from the above examples that SB is bridging the gap between basic and translational research. It is expected that with continuous technological advances in this field and with development of new programmable biological tools, SB has enormous potential to develop biomedical therapies to prevent and treat diseases caused by bacterial infections.

\footnotetext{
Abbreviations

AHL: N-acyl-L-homoserine lactone; CAl-1: Cholerae autoinducer-1; CDC: Centers for disease control and prevention; CRISPR: Clustered regularly interspaced short palindromic repeats; DNA: Deoxyribonucleic acid; GETR: Genome editing via targetrons and recombinases; GFP: Green fluorescent protein; iNOS: inducible nitric oxide synthase; MAGE: Multiplex automated genome engineering; mBTL: meta-bromo-thiolactone; MDR: Multidrug resistant; mRNA: messenger ribonucleic acid; MRSA: Methicillin resistant Staphylococcus aureus; NO: Nitric oxide; NRPS: Nonribosomal peptide synthetase; PCR: Polymerase chain reaction; PORTMAGE: portable multiplex automated genome engineering; PRSP: penicillin-resistant Streptococcus pneumoniae; QS: Quorum sensing: RBS: Ribosomal binding site; RNA: Ribonucleic acid; SB: Synthetic biology; VRE: Vancomycin-resistant enterococci; VRSA: Vancomycin-resistant Staphylococcus aureus; WHO: World Health Organization
}

\section{Funding}

This work was supported by the Department of Defense Chemical Biological Defense Program through the Defense Threat Reduction Agency (DTRA) under United States Army Medical Research Institute of Infectious Diseases (USAMRIID) project number 13267645. Opinions, interpretations, conclusions, and recommendations are those of the authors and are not necessarily endorsed by the U.S. Army. This manuscript was written when MK held an NRC Research Associateship award at USAMRIID. RTM was supported by Oak Ridge Institute for Science and Education (ORISE) Fellowship.

Availability of data and material

Not applicable.

Authors' contributions

MK, RTM, SR and RGP wrote the manuscript. All authors reviewed and approved the final manuscript before submission.

\section{Competing interests}

The authors declare that they have no competing interests.

\section{Consent for publication}

Not applicable.

Ethics approval and consent to participate

Not applicable.

\section{Endnotes}

Not applicable.

Received: 22 May 2016 Accepted: 28 October 2016

Published online: 04 November 2016

\section{References}

1. World Health Organization. Global action plan on antimicrobial resistance. Available http://www.who.int/iris/handle/10665/193736. World Health Organization. 2015.

2. Keasling JD. Synthetic biology and the development of tools for metabolic engineering. Metab Eng. 2012;14(3):189-95.

3. Yadav VG, De Mey M, Giaw Lim C, Kumaran Ajikumar P, Stephanopoulos G. The future of metabolic engineering and synthetic biology: Towards a systematic practice. Metab Eng. 2012;14(3):233-41.

4. Burbelo PD, Ching KH, Han BL, Klimavicz CM, ladarola MJ. Synthetic biology for translational research. Am J Transl Res. 2010;2(4):381-9.

5. Ruder WC, Lu T, Collins JJ. Synthetic biology moving into the clinic. Science. 2011;333(6047):1248-52.

6. Pei L. Synthetic biology approaches to combat antibiotic resistant bacteria. Microbiol Book Series \#4. 2013;1:724-7.

7. Nakashima N, Miyazaki K. Bacterial cellular engineering by genome editing and gene silencing. Int J Mol Sci. 2014;15(2):2773-93.

8. Feher T, Gyorfy Z. Microbial genome engineering for promoting health and understanding disease. Acta Biologica Szegediensis. 2015;59:169-87.

9. Gibson DG, Glass JI, Lartigue C, Noskov VN, Chuang RY, Algire MA, Benders GA, Montague MG, Ma L, Moodie MM, et al. Creation of a bacterial cell controlled by a chemically synthesized genome. Science. 2010;329(5987):52-6.

10. Stricker J, Cookson S, Bennett MR, Mather WH, Tsimring LS, Hasty J. A fast, robust and tunable synthetic gene oscillator. Nature. 2008;456(7221):516-9.

11. Lynch SA, Gill RT. Synthetic biology: new strategies for directing design. Metab Eng. 2012;14(3):205-11.

12. Moya A, Gil R, Latorre A, Pereto J, Pilar Garcillan-Barcia M, de la Cruz F. Toward minimal bacterial cells: evolution vs. design. FEMS Microbiol Rev. 2009;33(1):225-35.

13. Yu BJ, Kim JA, Ju HM, Choi SK, Hwang SJ, Park S, Kim E, Pan JG. Genomewide enrichment screening reveals multiple targets and resistance genes for triclosan in Escherichia coli. J Microbiol. 2012;50(5):785-91.

14. Kitagawa M, Ara T, Arifuzzaman M, loka-Nakamichi T, Inamoto E, Toyonaga $\mathrm{H}$, Mori H. Complete set of ORF clones of Escherichia coli ASKA library (a complete set of E. coli K-12 ORF archive): unique resources for biological research. DNA Res. 2005;12(5):291-9.

15. Kohanski MA, Dwyer DJ, Collins JJ. How antibiotics kill bacteria: from targets to networks. Nat Rev Microbiol. 2010;8(6):423-35.

16. Molina-Henares MA, de la Torre J, Garcia-Salamanca A, Molina-Henares AJ, Herrera MC, Ramos JL, Duque E. Identification of conditionally essential 
genes for growth of Pseudomonas putida KT2440 on minimal medium through the screening of a genome-wide mutant library. Environ Microbiol. 2010;12(6):1468-85.

17. Lin Y, Zhang RR. Putative essential and core-essential genes in Mycoplasma genomes. Sci Rep. 2011;1:53.

18. Zhang R, Ou HY, Zhang CT. DEG: a database of essential genes. Nucleic Acids Res. 2004;32(Database issue):D271-2.

19. Pines G, Freed EF, Winkler JD, Gill RT. Bacterial recombineering: genome engineering via phage-based homologous recombination. ACS Synth Biol. 2015;4(11):1176-85.

20. Murphy KC. Use of bacteriophage lambda recombination functions to promote gene replacement in Escherichia coli. J Bacteriol. 1998;180(8):2063-71.

21. Datsenko KA, Wanner BL. One-step inactivation of chromosomal genes in Escherichia coli K-12 using PCR products. Proc Natl Acad Sci U S A. 2000; 97(12):6640-5.

22. Sawitzke JA, Costantino N, Li XT, Thomason LC, Bubunenko M, Court C, Court DL. Probing cellular processes with oligo-mediated recombination and using the knowledge gained to optimize recombineering. J Mol Biol. 2011;407(1):45-59.

23. Lee DJ, Bingle LE, Heurlier K, Pallen MJ, Penn CW, Busby SJ, Hobman JL. Gene doctoring: a method for recombineering in laboratory and pathogenic Escherichia coli strains. BMC Microbiol. 2009:9:252.

24. Kuhlman TE, Cox EC. Site-specific chromosomal integration of large synthetic constructs. Nucleic Acids Res. 2010;38(6):e92.

25. Yang J, Sun B, Huang H, Jiang Y, Diao L, Chen B, Xu C, Wang X, Liu J, Jiang $W$, et al. High-efficiency scarless genetic modification in Escherichia coli by using lambda red recombination and I-Scel cleavage. Appl Environ Microbiol. 2014;80(13):3826-34.

26. Blank K, Hensel M, Gerlach RG. Rapid and highly efficient method for scarless mutagenesis within the Salmonella enterica chromosome. PLoS ONE. 2011;6(1):e15763.

27. van Kessel JC, Hatfull GF. Recombineering in mycobacterium tuberculosis. Nat Methods. 2007:4(2):147-52.

28. Gust B, Chandra G, Jakimowicz D, Yuqing T, Bruton CJ, Chater KF. Lambda red-mediated genetic manipulation of antibiotic-producing streptomyces. Adv Appl Microbiol. 2004;54:107-28.

29. Liang R, Liu J. Scarless and sequential gene modification in pseudomonas using PCR product flanked by short homology regions. BMC Microbiol. 2010;10:209

30. Schuster M, Lostroh CP, Ogi T, Greenberg EP. Identification, timing, and signal specificity of Pseudomonas aeruginosa quorum-controlled genes: a transcriptome analysis. J Bacteriol. 2003;185(7):2066-79.

31. O'Loughlin CT, Miller LC, Siryaporn A, Drescher K, Semmelhack MF, Bassler BL. A quorum-sensing inhibitor blocks Pseudomonas aeruginosa virulence and biofilm formation. Proc Natl Acad Sci U S A. 2013;110(44):17981-6.

32. Henard CA, Vazquez-Torres A. DksA-dependent resistance of Salmonella enterica serovar Typhimurium against the antimicrobial activity of inducible nitric oxide synthase. Infect Immun. 2012:80(4):1373-80.

33. Henard CA, Bourret TJ, Song M, Vazquez-Torres A. Control of redox balance by the stringent response regulatory protein promotes antioxidant defenses of Salmonella. J Biol Chem. 2010;285(47):36785-93.

34. Wang HH, Isaacs FJ, Carr PA, Sun ZZ, Xu G, Forest CR, Church GM Programming cells by multiplex genome engineering and accelerated evolution. Nature. 2009:460(7257):894-8.

35. Nyerges A, Csorgo B, Nagy I, Balint B, Bihari P, Lazar V, Apjok G, Umenhoffer K, Bogos B, Posfai G, et al. A highly precise and portable genome engineering method allows comparison of mutational effects across bacterial species. Proc Natl Acad Sci U S A. 2016;113(9):2502-7.

36. Lambowitz AM, Zimmerly S. Mobile group II introns. Annu Rev Genet. 2004:38:1-35

37. Enyeart PJ, Chirieleison SM, Dao MN, Perutka J, Quandt EM, Yao J, Whitt JT, Keatinge-Clay AT, Lambowitz AM, Ellington AD. Generalized bacterial genome editing using mobile group II introns and Cre-lox. Mol Syst Biol. 2013;9:685.

38. Heap JT, Pennington OJ, Cartman ST, Carter GP, Minton NP. The ClosTron: a universal gene knock-out system for the genus Clostridium. J Microbiol Methods. 2007;70(3):452-64.

39. Francis MB, Allen CA, Shrestha R, Sorg JA. Bile acid recognition by the Clostridium difficile germinant receptor, $\mathrm{CspC}$, is important for establishing infection. PLoS Pathog. 2013;9(5):e1003356.

40. Steen JA, Steen JA, Harrison P, Seemann T, Wilkie I, Harper M, Adler B, Boyce JD. Fis is essential for capsule production in Pasteurella multocida and regulates expression of other important virulence factors. PLoS Pathog. 2010;6(2):e1000750.

41. Kim E, Moore BS, Yoon YJ. Reinvigorating natural product combinatorial biosynthesis with synthetic biology. Nat Chem Biol. 2015;11(9):649-59.

42. Eustáquio AS, Gust B, Luft T, Li S-M, Chater KF, Heide L. Clorobiocin biosynthesis in streptomyces. Chem Biol. 2003;10(3):279-88.

43. Fowler Jr VG, Boucher HW, Corey GR, Abrutyn E, Karchmer AW, Rupp ME, Levine DP, Chambers HF, Tally FP, Vigliani GA, et al. Daptomycin versus standard therapy for bacteremia and endocarditis caused by Staphylococcus aureus. N Engl J Med. 2006;355(7):653-65.

44. Arbeit RD, Maki D, Tally FP, Campanaro E, Eisenstein Bl, Daptomycin, Investigators. The safety and efficacy of daptomycin for the treatment of complicated skin and skin-structure infections. Clin Infect Dis. 2004:38(12):1673-81.

45. Nguyen KT, Ritz D, Gu JQ, Alexander D, Chu M, Miao V, Brian P, Baltz RH. Combinatorial biosynthesis of novel antibiotics related to daptomycin. Proc Natl Acad Sci U S A. 2006;103(46):17462-7.

46. Arbues A, Aguilo JI, Gonzalo-Asensio J, Marinova D, Uranga S, Puentes E, Fernandez C, Parra A, Cardona PJ, Vilaplana C, et al. Construction, characterization and preclinical evaluation of MTBVAC, the first liveattenuated M. tuberculosis-based vaccine to enter clinical trials. Vaccine. 2013:31(42):4867-73.

47. Martin LB. Vaccines for typhoid fever and other salmonelloses. Curr Opin Infect Dis. 2012;25(5):489-99.

48. Ando H, Citorik R, Cleto S, Lemire S, Mimee M, Lu T. Synthetic Biology and Therapies for Infectious Diseases. In: Phoenix DA, Harris F, Dennison SR, editors. Novel Antimicrobial Agents and Strategies. Weinheim, Germany: Wiley VCH Verlag GmbH \& Co KGaA; 2014

49. Ranallo RT, Barnoy S, Thakkar S, Urick T, Venkatesan MM. Developing live Shigella vaccines using lambda Red recombineering. FEMS Immunol Med Microbiol. 2006:47(3):462-9.

50. Salehi A, Saadati M, Raoofian R, Bahador A, Doroudian M, Ahsanim Y, Hosseini M, Heidari M. I-Red-Recombineering Live Attenuated DipaD Shigella dysenteriae from Iranian Isolates as A Candidate of Vaccine. J Sci Islamic Republic Iran. 2012:23(1):21-8.

51. Barry EM, Pasetti MF, Sztein MB, Fasano A, Kotloff KL, Levine MM. Progress and pitfalls in Shigella vaccine research. Nat Rev Gastroenterol Hepatol. 2013;10(4):245-55

52. Citorik RJ, Mimee M, Lu TK. Bacteriophage-based synthetic biology for the study of infectious diseases. Curr Opin Microbiol. 2014;19:59-69.

53. Lu TK, Bowers J, Koeris MS. Advancing bacteriophage-based microbial diagnostics with synthetic biology. Trends Biotechnol. 2013;31(6):325-7.

54. Lu TK, Koeris MS. The next generation of bacteriophage therapy. Curr Opin Microbiol. 2011;14(5):524-31.

55. Citorik RJ, Mimee M, Lu TK. Sequence-specific antimicrobials using efficiently delivered RNA-guided nucleases. Nat Biotechnol. 2014;32(11):1141-5.

56. Bikard D, Euler CW, Jiang W, Nussenzweig PM, Goldberg GW, Duportet X, Fischetti VA, Marraffini LA. Exploiting CRISPR-Cas nucleases to produce sequence-specific antimicrobials. Nat Biotechnol. 2014;32(11):1146-50.

57. Yosef I, Manor M, Kiro R, Qimron U. Temperate and lytic bacteriophages programmed to sensitize and kill antibiotic-resistant bacteria. Proc Natl Acad Sci U S A. 2015:112(23):7267-72.

58. Slomovic S, Pardee K, Collins JJ. Synthetic biology devices for in vitro and in vivo diagnostics. Proc Natl Acad Sci U S A. 2015;112(47):14429-35.

59. Schukur L, Geering B, Charpin-El Hamri G, Fussenegger M. Implantable synthetic cytokine converter cells with AND-gate logic treat experimental psoriasis. Sci Transl Med. 2015;7(318):318ra201.

60. Waters CM, Bassler BL. Quorum sensing: cell-to-cell communication in bacteria. Annu Rev Cell Dev Biol. 2005;21:319-46.

61. Weiss R, Knight Jr T. Engineered communications for microbial robotics. DNA Comput. 2001;2054:1-16

62. Andrianantoandro E, Basu S, Karig DK, Weiss R. Synthetic biology: new engineering rules for an emerging discipline. Mol Syst Biol. 2006;2: 2006.0028.

63. Auslander S, Wieland M, Fussenegger M. Smart medication through combination of synthetic biology and cell microencapsulation. Metab Eng. 2012;14(3):252-60.

64. Weber W, Fussenegger M. Emerging biomedical applications of synthetic biology. Nat Rev Genet. 2012;13(1):21-35.

65. Duan F, March JC. Engineered bacterial communication prevents Vibrio cholerae virulence in an infant mouse model. Proc Natl Acad Sci U S A. 2010;107(25):11260-4 
66. Saeidi N, Wong CK, Lo TM, Nguyen HX, Ling H, Leong SS, Poh CL, Chang MW. Engineering microbes to sense and eradicate Pseudomonas aeruginosa, a human pathogen. Mol Syst Biol. 2011;7:521.

67. Gupta S, Bram EE, Weiss R. Genetically programmable pathogen sense and destroy. ACS Synth Biol. 2013;2(12):715-23.

68. Hwang IY, Tan MH, Koh E, Ho CL, Poh CL, Chang MW. Reprogramming microbes to be pathogen-seeking killers. ACS Synth Biol. 2014;3(4):228-37.

69. Green AA, Silver PA, Collins JJ, Yin P. Toehold switches: de-novo-designed regulators of gene expression. Cell. 2014;159(4):925-39.

70. Isaacs FJ, Dwyer DJ, Ding C, Pervouchine DD, Cantor CR, Collins JJ. Engineered riboregulators enable post-transcriptional control of gene expression. Nat Biotechnol. 2004;22(7):841-7.

71. Callura JM, Dwyer DJ, Isaacs FJ, Cantor CR, Collins JJ. Tracking, tuning, and terminating microbial physiology using synthetic riboregulators. Proc Natl Acad Sci U S A. 2010;107(36):15898-903.

72. Krishnamurthy M, Hennelly SP, Dale T, Starkenburg SR, Marti-Arbona R, Fox DT, Twary SN, Sanbonmatsu KY, Unkefer CJ. Tunable Riboregulator Switches for Post-transcriptional Control of Gene Expression. ACS Synth Biol. 2015; 4(12):1326-34

73. Pardee K, Green AA, Ferrante T, Cameron DE, DaleyKeyser A, Yin P, Collins JJ. Paper-based synthetic gene networks. Cell. 2014;159(4):940-54.

74. Challis GL. Mining microbial genomes for new natural products and biosynthetic pathways. Microbiology. 2008;154(Pt 6):1555-69.

75. Hutchison CA, Chuang 3rd RY, Noskov VN, Assad-Garcia N, Deerinck TJ, Ellisman MH, Gill J, Kannan K, Karas BJ, Ma L, et al. Design and synthesis of a minimal bacterial genome. Science. 2016;351(6280):aad6253.

76. Nielsen AA, Der BS, Shin J, Vaidyanathan P, Paralanov V, Strychalski EA, Ross D, Densmore D, Voigt CA. Genetic circuit design automation. Science. 2016; 352(6281):aac7341.

77. Chan CT, Lee JW, Cameron DE, Bashor CJ, Collins JJ. 'Deadman' and 'Passcode' microbial kill switches for bacterial containment. Nat Chem Biol. 2016;12(2):82-6.

78. Kumar S, Smith KP, Floyd JL, Varela MF. Cloning and molecular analysis of a mannitol operon of phosphoenolpyruvate-dependent phosphotransferase (PTS) type from Vibrio cholerae O395. Arch Microbiol. 2011;193(3):201-8.

79. Palonen E, Lindstrom M, Karttunen R, Somervuo P, Korkeala H. Expression of signal transduction system encoding genes of Yersinia pseudotuberculosis IP32953 at 28 degrees C and 3 degrees C. PLoS ONE. 2011;6(9):e25063.

80. Yao J, Zhong J, Fang Y, Geisinger E, Novick RP, Lambowitz AM. Use of targetrons to disrupt essential and nonessential genes in staphylococcus aureus reveals temperature sensitivity of LI.LtrB group II intron splicing. RNA. 2006;12(7):1271-81.

81. Ji Y, Zhang B, Van SF, Horn, Warren P, Woodnutt G, Burnham MK, Rosenberg M. Identification of critical staphylococcal genes using conditional phenotypes generated by antisense RNA. Science. 2001; 293(5538):2266-9.

82. Uguru GC, Mondhe M, Goh S, Hesketh A, Bibb MJ, Good L, Stach JE. Synthetic RNA silencing of actinorhodin biosynthesis in streptomyces coelicolor A3(2). PLoS ONE. 2013;8(6):e67509.

\section{Submit your next manuscript to BioMed Central and we will help you at every step:}

- We accept pre-submission inquiries

- Our selector tool helps you to find the most relevant journal

- We provide round the clock customer support

- Convenient online submission

- Thorough peer review

- Inclusion in PubMed and all major indexing services

- Maximum visibility for your research

Submit your manuscript at www.biomedcentral.com/submit

) Biomed Central 